\title{
Traitement de l'alcoolisme par le baclofène
}

\section{Baclofen for the treatment of alcoholism}

\author{
R. de Beaurepaire \\ (C) Springer-Verlag France 2011
}

Résumé Des données cliniques et des témoignages de patients qui indiquent une efficacité du baclofène dans le traitement de l'alcoolisme se sont multipliés au cours de ces deux dernières années, mais beaucoup de prescripteurs sont toujours très réticents à l'utiliser. Pourtant, l'alcoolisme est une maladie mortelle pour laquelle n'existe aucun traitement véritablement efficace, en dehors du baclofène. Dans cet article, l'auteur rapporte son expérience de prescripteur de baclofène, avec une analyse des obstacles potentiels susceptibles de limiter son efficacité. Une approche des mécanismes biologiques par lesquels pourrait agir le baclofène est aussi proposée. Pour citer cette revue : Psychiatr. Sci. Hum. Neurosci. 9 (2011).

Mots clés Addiction · Anxiété · Indifférence · Somnolence · Amygdale

\begin{abstract}
Clinical data and patients' accounts have provided accumulating evidence supporting the efficacy of baclofen in the treatment of alcoholism. However, alcohologists remain reluctant to prescribe baclofen. It should be remembered that alcoholism is a lethal illness for which no efficacious treatment exists, except baclofen. The author of the present paper relates his experience with the prescription of baclofen, and analyses the potential obstacles that may limit its efficacy. A biological approach of the mechanisms by which baclofen may act is also proposed. To cite this journal: Psychiatr. Sci. Hum. Neurosci. 9 (2011).
\end{abstract}

Keywords Addiction · Anxiety · Indifference $\cdot$ Somnolence Amygdala

L'alcoolisme est une maladie dévastatrice qui tue environ 60000 personnes par an en France, ce qui fait 120 personnes par jour. La mortalité liée à l'alcoolisme inclut aussi bien les cirrhoses que les accidents de la circulation imputables à

R. de Beaurepaire $(\bowtie)$

Groupe hospitalier Paul Guiraud, F-94806 Villejuif, France

e-mail : debeaurepaire@wanadoo.fr l'alcool. En termes d'économie de la santé, le coût de l'alcoolisme est énorme. En termes de souffrances psychologiques, individuelles et familiales, le coût est tout aussi grand. Il existe plusieurs maladies autres que l'alcoolisme qui sont aussi très répandues et coûteuses, par exemple le diabète ou la schizophrénie, mais ces maladies se soignent, elles ont des traitements spécifiques et efficaces. Or, il n'y a pas de traitement de l'alcoolisme. Que l'on ne nous dise pas qu'il y a des traitements efficaces pour l'alcoolisme, ce n'est pas vrai. Un médicament tel que la naltrexone a peut-être une petite efficacité à court terme chez certains patients, mais son efficacité à plus long terme, à un an par exemple, est nulle [7]. « Le nihilisme thérapeutique s'applique tout aussi bien à l'acamprosate » dit Stahl, le pape actuel de la psychopharmacologie [15]. Cela n'empêche pas qu'il existe en France d'énormes infrastructures pour la prise en charge des alcooliques. Elles se sont progressivement mises en place au cours de ces 40 dernières années, avec les centres d'hygiène alimentaire et divers autres centres de consultation, une spécialité médicale — l'alcoologie — , des médecins alcoologues, des associations subventionnées par de nombreux organismes, des centres de cure, des centres de postcure, des techniques multiples de prise en charge, des sociétés savantes, des écoles, des journaux, des colloques, des comités, des rapports, des carrières, etc., pour des résultats dérisoires. Un énorme appareil, huilé, institutionnalisé, ramifié, ronronnant, lucratif pour certains et très coûteux pour la société. Assez justifié vu le nombre d'alcooliques en France, l'énormité des infrastructures apparaissant parfaitement proportionnelle à l'énormité de la demande. Mais en termes d'efficacité ? On peut avoir l'impression que l'énormité de ces infrastructures est surtout proportionnelle à leur inefficacité sur le plan thérapeutique. On dira, avec un peu de mauvaise foi, qu'elles pourraient n'être là que pour faire comme si. Comme si on était capable de soigner l'alcoolisme. On ne l'est pas.

Et puis est arrivé un médicament qui guérit l'alcoolisme. Personne ne l'attendait. Et il est très vite apparu que personne n'en voulait. Comme si un traitement efficace risquait de mettre en péril la grosse machine à soigner les alcooliques qui ne sert qu'à faire comme si. 
Le médicament s'appelle le baclofène. C'est un myorelaxant commercialisé en 1972 pour traiter les spasmes musculaires que l'on observe dans certaines maladies neurologiques, telles que la sclérose en plaques. Un médicament bien connu des neurologues, qui en ont même développé une forme pouvant être injectée directement dans le cerveau, la forme intrathécale. On injecte donc du baclofène directement au contact du cerveau, et, apparemment, cela ne pose pas de problème majeur de tolérance (mais cela expose à des complications infectieuses, ce qui fait que cette voie est peu utilisée). Les neurologues prescrivent donc du baclofène depuis près de 40 ans, couramment à des enfants, parfois directement dans le cerveau. Les neurologues ont aussi observé le fait que pour avoir une réelle efficacité, il fallait souvent donner du baclofène à des doses bien supérieures à celles préconisées (dans l'autorisation de mise sur le marché, ou AMM, en France, les doses maximales préconisées sont de $75 \mathrm{mg} / \mathrm{j}$ en ambulatoire et $120 \mathrm{mg} / \mathrm{j}$ chez les personnes hospitalisées). Le baclofène est prescrit par les neurologues à doses progressivement croissantes, jusqu'à obtention de l'effet antispasmodique. Les neurologues donnent ainsi couramment et depuis longtemps du baclofène à des doses de 200 ou $300 \mathrm{mg} / \mathrm{j}$, sans que personne n'y trouve rien à redire [14].

\section{Effets thérapeutiques du baclofène dans l'alcoolisme}

Les effets thérapeutiques du baclofène dans l'alcoolisme ont été découverts par un médecin français, Ameisen [2]. Pour le récit de cette extraordinaire découverte, on renvoie le lecteur au livre d'Ameisen, Le dernier verre, publié en France en 2008 [4]. Brièvement, Ameisen était dépendant à l'alcool et il a trouvé dans la littérature scientifique des articles qui montraient que quand on donne du baclofène à un rat rendu dépendant aux drogues, cette dépendance cesse [13]. La dépendance aux drogues chez le rat cesse à des doses de l'ordre de 3 ou $4 \mathrm{mg} / \mathrm{kg}$ de baclofène, soit plus de $200 \mathrm{mg}$ chez un homme de $70 \mathrm{~kg}$, et Ameisen a cessé d'être dépendant à l'alcool à $270 \mathrm{mg} / \mathrm{j}$ de baclofène. À cette dose de $270 \mathrm{mg}$, Ameisen s'est rendu compte qu'il était devenu totalement indifférent à l'alcool. Cela aurait pu n'être qu'un cas clinique isolé, mais cela ne l'a pas été. Depuis lors, des dizaines de médecins ont rapporté un effet similaire, l'indifférence vis-à-vis de l'alcool, certains disent la suppression du craving (mais on verra plus loin que ce n'est peut-être pas l'expression exacte), chez les patients à qui ils ont prescrit du baclofène à dose progressivement croissante, sans limite supérieure. Plusieurs centaines de cas sont aujourd'hui rapportés (pas tous publiés, mais pour l'essentiel rapportés ici ou là sur des forums Internet, ou par des communications personnelles à Ameisen et à moi-même).
Ameisen et moi-même avons publié une courte série de 60 cas de patients suivis pendant trois mois [5]. Nous avons, depuis, traité un beaucoup plus grand nombre de patients, mais sur le plan statistique, les chiffres n'ont pas beaucoup changé. Des collègues qui ont actuellement des séries de plusieurs dizaines de patients nous ont dit avoir aussi trouvé des chiffres similaires. Les chiffres de notre étude publiée, qui pourraient donc être assez représentatifs de plus grandes séries de patients, sont les suivants (à trois mois). Près de $90 \%$ des patients ont éprouvé les effets suppresseurs du baclofène sur l'envie de boire. La majorité ont totalement cessé de boire, ou boivent encore un verre de temps en temps, généralement sans finir le verre. Néanmoins, un certain nombre de patients, qui disaient clairement éprouver les effets suppresseurs du baclofène sur leur envie de boire, ont eu beaucoup plus de difficultés à arrêter de boire, et certains n'ont pas arrêté de boire, même si leur consommation globale d'alcool a beaucoup diminué (diminution de plus de $50 \%$ comparativement à la consommation avant baclofène). Nous avons beaucoup réfléchi et discuté le cas de ce groupe de patients que nous avons qualifiés de « demiguéris ». Il semblerait que ces patients aient été incapables d'arrêter de boire pour trois raisons principales : une motivation insuffisante, une pathologie psychiatrique concomitante et une intolérance aux effets indésirables du baclofène.

Une réelle motivation pour arrêter de boire apparaît comme une condition préalable indispensable pour que le baclofène soit efficace. Un assez grand nombre de patients que nous avons soignés nous étaient « amenés », pour ne pas dire «poussés », par un conjoint, ou des parents, ou un confrère, voire un employeur ou des services sociaux, alors même que le désir d'arrêter de boire n'était pas du tout évident chez les patients eux-mêmes. Il est apparu que l'alcool pouvait avoir une fonction terriblement importante chez certaines personnes. Une fonction souvent identitaire, telle que certaines personnes donnaient l'impression qu'elles «n'existeraient plus » si elles arrêtaient de boire. Parfois une fonction antidépressive, certaines personnes disant qu'elles ne pouvaient sortir d'un état de profonde tristesse, ou de repli complet sur soi, que grâce à l'alcool. Ou une fonction clairement hédonique, peut-être pas très éloignée de la fonction antidépressive précédente, mais quand même différente, où les patients disent aimer la fête, ne vivre que pour faire la fête, pour "s'éclater ", trouvant insupportablement ennuyeuse la vie sans alcool. Chez d'autres, l'alcool a manifestement une fonction transactionnelle vis-à-vis de certains proches, la mère souvent, où la prise destructrice d'alcool est l'expression d'une souffrance, d'un refus, d'une immaturité, certains pourront dire d'un œdipe non résolu, dans une systémique bloquée. On pourrait donner d'autres illustrations de ces fonctions de l'alcool qui rendent inopérants les effets réducteurs du baclofène sur l'appétence pour l'alcool, c'est-à-dire qui font que beaucoup de patients ne peuvent 
pas avoir de motivation suffisante pour arrêter de boire, la prise d'alcool étant trop importante, pour ne pas dire « vitale », chez eux.

Plus de la moitié des patients à qui nous avons prescrit du baclofène souffraient de troubles mentaux. En général, les patients connaissaient leur maladie psychiatrique et étaient suivis par un psychiatre. Les troubles anxieux et dépressifs étaient les diagnostics les plus fréquents, un bon nombre de patients présentaient un trouble bipolaire, et nous avons diagnostiqué de très nombreux cas de trouble de la personnalité. La plupart des patients prenaient un traitement correspondant à leur trouble, le plus souvent ils amenaient une ordonnance. Nous n'avons changé aucun traitement, ni même conseillé de changement. Notre attitude a toujours été de dire que le traitement par le baclofène est totalement indépendant des autres traitements (psychiatriques et somatiques), et qu'en aucun cas, même pas par la suggestion, nous n'intervenons dans les traitements en cours. Néanmoins, nous avons toujours lu avec chaque patient une note d'information mentionnant les précautions d'emploi $\mathrm{du}$ baclofène, parmi lesquelles figuraient des interactions potentielles du baclofène avec certains psychotropes. Nous avons aussi systématiquement appelé les médecins traitants pour les informer de la prescription de baclofène. L'analyse des résultats a montré que l'existence d'une maladie psychiatrique pouvait être corrélée à une moindre efficacité du baclofène (statistiquement très significatif). On pourrait disserter longuement sur les raisons de cette corrélation. On rappelle qu'il existe incontestablement une dimension transversale de « maladie de la volonté » dans toutes les maladies psychiatriques. On a vu que pour obtenir un effet optimal du baclofène, il était essentiel que les patients soient motivés pour arrêter. La motivation des patients souffrant des troubles mentaux peut être diminuée pour toutes sortes de raisons ou circonstances : pour des raisons intrinsèques à la maladie («maladies de la volonté »), selon le type de maladie (effet anxiolytique de l'alcool chez les anxieux, ou antidépresseur chez les déprimés), du fait de troubles de la personnalité associés (par exemple, alternances idéalisation/disqualification du baclofène chez les borderline), du fait de l'observance du traitement (connue pour être particulièrement mauvaise chez les malades mentaux en général), ou encore du fait des bénéfices secondaires liés à l'alcoolisme (exposition d'une souffrance pour mobiliser l'entourage, etc.). Dans ces conditions, il n'est peut-être pas très étonnant d'avoir observé une moindre efficacité du baclofène chez les patients souffrant de troubles mentaux.

Les effets secondaires du baclofène ont constitué un autre obstacle à son efficacité. Dans l'étude sus-mentionnée portant sur 60 cas à trois mois, nous avions rapporté que plus de $80 \%$ des patients avaient rapporté des effets indésirables. Avec un recul de près de deux ans, et plusieurs centaines de cas analysés, ce pourcentage reste approximativement le même. Même si ces effets indésirables du baclofène sont tous bénins (c'est-à-dire ne mettent jamais en jeu le pronostic vital), certains, du fait de leur intensité, ont amené des patients à arrêter leur traitement, ou à ne pas augmenter les doses suffisamment pour obtenir un effet thérapeutique. La fatigue est l'effet secondaire le plus fréquent du baclofène. Cette fatigue peut être très gênante quand elle est intense. Elle prend parfois la forme de véritables attaques de sommeil, et peut s'accompagner de vertiges et de malaises. Le baclofène à faible dose produit parfois des états subconfusionnels qui peuvent inquiéter beaucoup les patients. À fortes doses, nous avons observé plusieurs cas de véritables syndromes confusionnels, mais chaque fois les fortes doses de baclofène étaient associées à la consommation de fortes doses d'alcool et de benzodiazépines, de telle sorte qu'il a été difficile d'incriminer le seul baclofène. Une crise d'épilepsie a aussi été observée chez une patiente qui n'avait pas d'antécédent d'épilepsie. Certains patients ont présenté une incontinence, d'autres des vomissements répétés, et chez deux patients, des diarrhées persistantes. Ces événements indésirables très gênants (diarrhée, vomissements, incontinence, épilepsie, état confusionnel, fatigue intense, attaques de sommeil) n'ont concerné qu'une petite minorité de patients, mais ils ont amené certains à arrêter le baclofène. Or, ces événements indésirables sont en règle gérables. La plupart sont accessibles à un traitement spécifique (antidiarrhéique, antiémétique, etc.), mais surtout ils sont gérables en adaptant la conduite du traitement par le baclofène. Il est rare que ces effets indésirables surviennent d'emblée, à la prise du premier comprimé de baclofène. Ils surviennent en règle à une certaine dose, au cours de l'augmentation des doses par paliers. Lors de la survenue d'un événement indésirable très gênant, l'attitude conseillée est de proposer au patient de diminuer les doses de baclofène, en repassant au palier inférieur de dose, celui qui ne produisait pas d'effets indésirables, et de maintenir un certain temps à cette dose. Puis de réaugmenter les doses très lentement et prudemment, par exemple d'un comprimé par semaine. On arrive généralement ainsi à passer le cap de beaucoup d'effets indésirables, qui ne réapparaissent pas quand on ralentit la progression des doses. Il est bien connu que beaucoup des effets indésirables du baclofène (c'est mentionné dans le Vidal) sont liés à une augmentation trop rapide des doses. Chez les patients qui arrêtent leur traitement en alléguant des effets indésirables, la question de leur motivation pour arrêter de boire se pose d'une façon évidente. Nous avons d'ailleurs observé des patients qui ont arrêté leur traitement en alléguant des effets indésirables qui nous semblaient très mineurs. Autrement dit, c'est la motivation des patients pour arrêter de boire qui est en question dans ces cas. Les patients suffisamment motivés ne se découragent pas pour un effet indésirable, si gênant soit-il, et suivent avec beaucoup d'attention les suggestions qui leur sont faites de diminuer les doses pendant un 
certain temps, puis de réessayer une augmentation très lente des doses. Il faut aussi noter, pour introduire le paragraphe suivant, que nous n'avons observé aucune addiction au baclofène (sauf peut-être chez deux patients, mais l'addiction au baclofène était discutable chez eux dans le sens où ils étaient addictifs à pratiquement tous les psychotropes à leur portée).

\section{Mécanisme d'action du baclofène}

Le baclofène est un agoniste des récepteurs gaba-B (il existe dans le cerveau deux types de récepteurs au gaba, le gaba-A et le gaba-B). Le baclofène diffère ainsi des benzodiazépines utilisées dans le sevrage alcoolique, qui sont principalement des agonistes du récepteur gaba-A. Il n'existe pas d'autre agoniste des récepteurs gaba-B disponibles sur le marché. À l'exception du GHB (acide gamma-hydroxybutyrique), non commercialisé en France, mais commercialisé dans d'autres pays européens, donc potentiellement disponible illégalement en France. Le problème du GHB est qu'il a des effets indésirables qui peuvent être dangereux, c'est un anesthésiant, un euphorisant, certains sportifs l'utilisent pour se doper, et il est addictogène. Sa célébrité lui vient surtout de ce qu'il est le "médicament des violeurs ». Le GHB existe à l'état naturel dans l'organisme, en petites quantités, et il a des récepteurs spécifiques dans le cerveau. Il a été montré que le GHB diminue le craving pour l'alcool chez l'homme [6]. Ses effets physiologiques chez l'animal ressemblent beaucoup à ceux du baclofène, et l'activation qu'il produit dans les diverses régions du cerveau ressemble beaucoup à celle du baclofène, en particulier dans l'hypothalamus et l'amygdale [16]. En 2007, Ameisen a proposé l'hypothèse selon laquelle l'alcoolisme pourrait être lié à un défaut de production endogène de GHB [3]. L'argumentation d'Ameisen repose sur les éléments suivants : l'activité agoniste du GHB sur les récepteurs gaba-B, son effet anticraving bien démontré, et le fait que le GHB, qui est sédatif, anxiolytique et myorelaxant, pourrait avoir un effet thérapeutique spécifique sur les principaux symptômes de la dépendance, qui sont l'insomnie, l'anxiété et la tension musculaire. L'apport exogène de baclofène pouvant ainsi, selon cette hypothèse, pallier le manque de stimulation endogène des récepteurs gaba-B par le GHB. À la différence du GHB, le baclofène agirait sélectivement sur les récepteurs gaba-B, qui ne seraient pas impliqués dans le développement d'une dépendance, alors que le GHB a de nombreux sites d'action, dont certains sont susceptibles de participer à la mise en place d'une dépendance.

Il n'existe donc en France qu'un seul agoniste sélectif du récepteur gaba-B disponible sur le marché, le baclofène. Cet agoniste supprime l'envie de boire chez les alcooliques, ce qui implique logiquement que le l'envie de boire chez les alcooliques passe par le récepteur gaba-B (un défaut de stimulation de ce récepteur). Cela soulève la question des liens biologiques, et des différences, entre l'envie de boire et le craving. En effet, on note que, curieusement [16], le baclofène n'a pas d'effet activateur dans la région du cerveau qui est classiquement la plus impliquée dans le craving le système dopaminergique de plaisir mésoaccumbens, qui est le site d'action de toutes les substances qui ont des propriétés addictives. Cela devrait impliquer que la suppression de l'envie de boire produite par le baclofène, ou l'indifférence à l'alcool est le résultat de son action sur un système indépendant du système dopaminergique de plaisir. Ce qui peut être une surprise dans le sens où les neurobiologistes considèrent habituellement que ce système de plaisir a une position centrale dans la physiologie de la dépendance à l'alcool et dans son traitement [11]. C'est d'ailleurs sur ce système que semblent agir la naltrexone et l'acamprosate. On sait néanmoins que la mise en place de la dépendance passe par plusieurs étapes, la première mettant en jeu spécifiquement le système dopaminergique mésoaccumbens, et les étapes suivantes consistant en ce que l'on appelle la « transition du système addictif », qui fait que l'envie de se procurer des substances devient largement dépendante d'autres structures cérébrales telles que l'amygdale en interaction avec certaines régions du cortex préfrontal médian (orbitaire et cingulaire). Une fois mis en place, le système addictif reposerait donc sur deux systèmes biologiques, un système de plaisir modifié (avec en particulier des modifications des récepteurs dopaminergiques D2 dans le striatum, responsables du craving) et un système amygdalocortical modifié dans sa réactivité (et responsable d'autres aspects de la dépendance). Ce dernier système est connu pour être impliqué dans la mémorisation des expériences de plaisir ou de souffrance et dans leur rappel en présence d'indices environnementaux qui ont une relation avec ces expériences. La présence de récepteurs gaba-B dans l'amygdale incite à soulever l'hypothèse selon laquelle ce serait en agissant à ce niveau que le baclofène exerce son effet thérapeutique dans l'alcoolisme. Et il est intéressant, dans ce contexte, de signaler que la mémoire est largement dépendante de mécanismes glutamatergiques dans l'amygdale, et que le baclofène, en agissant sur les récepteurs gaba-B, déclenche une cascade d'événements qui conduisent à une inhibition glutamatergique [12].

L'amygdale est une des régions du cerveau les plus impliquées dans l'anxiété. Les récepteurs gaba-B dans le noyau basolatéral de l'amygdale ont un rôle dans le contrôle des états anxieux. Quel que soit le traitement médicamenteux qu'ils ont utilisé (en dehors du baclofène), les patients qui ont arrêté de boire grâce à un traitement et à la volonté rechutent dans l'année dans $80 \%$ des cas (un pourcentage comparable à celui obtenu avec un placebo). Il est assez couramment admis que l'anxiété, le stress et les stimuli conditionnés sont les causes les plus courantes de 
rechute [9]. Sur le plan neurobiologique, la prise chronique d'alcool aurait un effet stimulant sur les systèmes gabaergiques et inhibiteur sur les systèmes glutamatergiques, et les rechutes seraient en rapport avec un déséquilibre entre ces deux systèmes durant le sevrage en alcool, où les systèmes gabaergiques sont insuffisamment stimulés et où les systèmes glutamatergiques deviennent hyperactifs. Les rechutes pourraient survenir longtemps après le sevrage en alcool du fait de réponses mnésiques conditionnées, qui font intervenir le gaba et le glutamate dans l'amygdale. Les circonstances sont l'exposition à un certain nombre de situations, telles qu'un indice de la consommation d'alcool présent dans l'environnement, ou une angoisse associée à un conflit, ou à un sentiment d'abandon et de solitude, qui réactivent une souffrance du manque et produisent un besoin impulsif de consommer. L'angoisse est au centre de la rechute, toujours intimement liée à un déséquilibre entre ces deux neurotransmetteurs. Des études montrent d'ailleurs que c'est plus la présence d'indices contextuels de consommation d'alcool (et le besoin de calmer l'angoisse associée à ces indices) que le phénomène de craving lui-même qui est associé aux rechutes. Dans ces conditions, il pourrait être plus approprié de rapporter les effets du baclofène à ses effets anxiolytiques et à sa capacité à rendre indifférent à l'alcool, qu'à un effet « suppresseur du craving ». L'angoisse pourrait avoir pour corollaire la contrainte, ou le concernement (l'angoisse implique que l'on est pathologiquement concerné par quelque chose), des phénomènes qui seraient plus au centre de la rechute que le craving. L'effet du baclofène serait de produire un état de non-concernement pour l'alcool et ses indices contextuels, autrement dit une indifférence vis-à-vis de l'alcool, contemporaine d'un effet anxiolytique. La question à résoudre devient celle de savoir si le baclofène rend indifférent à l'alcool parce qu'il a des effets anxiolytiques, ou si ses effets anxiolytiques sont indépendants de sa capacité à rendre indifférent à l'alcool. Autrement dit, pour comprendre le mode d'action du baclofène, il sera nécessaire de disséquer les différents mécanismes, ou différentes composantes, qui participent à la rechute, qui sont le craving, l'anxiété et les phénomènes d'indifférence/ concernement vis-à-vis de l'alcool.

Des effets anxiolytiques du baclofène ont été retrouvés dans certaines formes d'anxiété chez l'homme, en particulier dans les états stress post-traumatiques [8] (nous n'avons pas trouvé de publication montrant que le baclofène aurait un effet thérapeutique dans d'autres formes d'anxiété). Chez l'animal, les effets anxiolytiques du baclofène sont contrastés et pas toujours évidents (pour revue, voir [10]). Dans la série de patients que nous avons traités, les effets anxiolytiques du baclofène étaient évidents chez beaucoup de patients, mais pas chez tous. Certains ont même rapporté se sentir moins bien, plus anxieux et irritables, avec le baclofène. Ces derniers patients constituent néanmoins une mino- rité. La majorité des patients a éprouvé un sentiment de bienêtre sous baclofène, beaucoup ont parlé de sérénité, certains ont présenté des symptômes manifestes d'euphorie légère (ou d'hypomanie). Dans ce cadre des troubles de l'humeur, il faut aussi signaler que certains patients ont présenté des symptômes dépressifs (dont il est difficile de dire s'ils sont attribuables au baclofène ou à d'autres éléments chez ces patients qui ont souvent de lourds antécédents de dépression). Ce qui ressort de ces données cliniques assez contrastées, c'est que les effets du baclofène sont très différents d'un sujet à l'autre sur les plans de l'anxiété et de l'humeur. En revanche, ce qui a toujours été très constant, c'est la mise en place d'une indifférence vis-à-vis de l'alcool (même si des différences interindividuelles ont été observées, comme décrit plus haut dans cet article). Si bien que ce qui pourrait faire l'unité dans les effets du baclofène, c'est plus l'état d'indifférence vis-à-vis de l'alcool que son effet anxiolytique. L'amygdale est connue pour son implication dans l'anxiété, mais aussi pour créer un état de nonreconnaissance, ou d'indifférence, à l'égard de ce qui fait naturellement peur. Une lésion bilatérale de l'amygdale, comme dans la maladie d'Urbach-Wiethe, produit des anomalies de la reconnaissance des expressions faciales, avec une non-reconnaissance des stimuli socialement effrayants [1]. Un état d'indifférence vis-à-vis des stimuli effrayants peut être interprété comme une forme d'anxiolyse. Mais il y a certainement lieu aussi de distinguer anxiolyse et indifférence. Un large champ de recherches est donc ouvert par le baclofène : sa capacité à créer un état d'indifférence vis-à-vis de l'alcool est-elle liée à son action sur l'amygdale ? Quel est le rôle d'autres régions du cerveau connues pour être impliquées dans l'anxiété et dans lesquelles existent des récepteurs gaba-B (raphé, noyau du lit de la strie terminale, hypothalamus) ? Est-il bien vrai que le baclofène n'agit pas sur le système de plaisir (certaines données expérimentales tendraient à montrer qu'il agit sur ce système) ? Quels sont les liens entre anxiolyse et indifférence ? Quel est le rôle des récepteurs gaba-B dans l'amygdale dans cette indifférence ? Et bien d'autres questions.

\section{Conclusion}

Le baclofène apparaît cliniquement comme un médicament d'une extraordinaire efficacité dans le traitement de l'alcoolisme. Que les alcoologues le veuillent ou non, il faudra bien un jour qu'ils le prescrivent. Il est très intéressant d'analyser les raisons qui font que le baclofène est d'une efficacité totale chez plus de la moitié des alcooliques, mais que chez un bon tiers d'entre eux l'effet thérapeutique du baclofène, même s'il est bien réel, reste incomplet. L'analyse des causes de ces « demi-succès » montre que tous les patients dépendants à l'alcool ne le sont pas pour les mêmes raisons, et que 
certaines de ces raisons peuvent constituer des obstacles à l'efficacité du baclofène. L'étude des mécanismes biologiques par lesquels agit potentiellement le baclofène ouvre sur un nombre considérable de questions, qui mettent en évidence la complexité et les multiples facettes du phénomène de la dépendance.

\section{Références}

1. Adolphs R, Tranel D, Damasio AR (1998) The human amygdala in social judgment. Nature 393(6684):470-4

2. Ameisen $O$ (2005) Complete and prolonged suppression of symptoms and consequences of alcohol-dependence using highdose baclofen: a self-case report of a physician. Alcohol Alcohol 40:147-50

3. Ameisen O (2007) Gamma-hydroxybutyrate (GHB)-deficiency in alcohol-dependence? Alcohol Alcohol 42:506

4. Ameisen O (2008) Le dernier verre. Denoël, Paris

5. Ameisen O, de Beaurepaire R (2010) Suppression de la dépendance à l'alcool et de la consommation d'alcool par le baclofène à haute dose : un essai en ouvert. Ann Med Psychol 168:159-62

6. Caputo F, Addolorato G, Lorenzini F, et al (2003) Gammahydroxybutyric acid versus naltrexone in maintaining alcohol abstinence: an open randomized comparative study. Drug Alcohol Depend 70:85-91
7. Chick J (2002) Naltrexone for 3 or 12 months in addition to psychosocial counselling did not reduce drinking in alcohol dependence. Evid Based Ment Health 5:80

8. Drake RG, Davis LL, Cates ME, et al (2003) Baclofen treatment for chronic posttraumatic stress disorder. Ann Pharmacother $37: 1177-81$

9. Everitt BJ, Robbins TW (2005) Neural systems of reinforcement for drug addiction: from actions to habits to compulsion. Nat Neurosci 8:1481-9

10. Frankowska M, Filip M, Przegalinski E (2007) Effects of GABAB receptor ligands in animal tests of depression and anxiety. Pharmacol Rep 59:645-55

11. Heinz A, Beck A, Grüsser SM, et al (2009) Identifying the neural circuitry of alcohol craving and relapse vulnerability. Addict Biol $14: 108-18$

12. Pan BX, Dong Y, Ito W, et al (2009) Selective gating of glutamatergic inputs to excitatory neurons of amygdala by presynaptic GABAB receptor. Neuron 61:917-29

13. Roberts DC, Andrews MM (1997) Baclofen supression of cocaine self-administration: demonstration using a discrete trials procedure. Physiopharmacology (Berl) 131:271-7

14. Smith CR, LaRocca NG, Giesser BS, Scheinberg LC (1991) High-dose oral baclofen: experience in patients with multiple sclerosis. Neurology 41:1829-31

15. Stahl SM (2008) Stahl's essential psychopharmacology. Cambridge University Press, New York

16. Van Nieuwenhuijzen PS, McGregor IS, Hunt GE (2009) The distribution of gamma-hydroxybutyrate-induced Fos expression in rat brain: comparison with baclofen. Neuroscience 158:441-55 